\title{
Malignant Spermatic Cord Neoplasm
}

National Cancer Institute

\section{Source}

National Cancer Institute. Malignant Spermatic Cord Neoplasm. NCI Thesaurus. Code C3559.

A primary or metastatic malignant neoplasm that affects the spermatic cord. 